Space Science Reviews manuscript No.

(will be inserted by the editor)

\title{
Water in extrasolar planets and implications for habitability
}

\author{
Lena Noack · Ignas Snellen • Heike \\ Rauer
}

Received: date / Accepted: date

\begin{abstract}
Exoplanet detection missions have found thousands of planets or planet candidates outside of the Solar System - some of which are in the habitable zone, where liquid water is possible at the surface. We give an overview of the recent progress in observations of water-rich exoplanets, detection of water in the atmosphere of gas giants and less-massive targets, and modelling of the interior and evolution of water layers in exoplanets. We summarise the possible habitability of water-rich planets and discuss the potential of future missions and telescopes towards the detection of water in the atmosphere of low-mass exoplanets or on their surface.
\end{abstract}

Keywords exoplanets · water · detection · atmosphere spectroscopy · habitability

\section{Introduction}

Water is abundant not only in the Solar System, but in the universe in general. The search for water on exoplanets is mainly driven by its implications for the potential habitability of these planets, since life as we know it from Earth needs

\section{Noack}

Royal Observatory of Belgium, Brussels, Belgium

Present address: Free University Berlin, Germany

Tel.: +49-30-838-63694

Fax: +49-30-8384-63694

E-mail: lena.noack@fu-berlin.de

H. Rauer

German Aerospace Center, Institute of Planetary Research, Berlin, Germany, and

Center for Astronomy and Astrophysics, TU Berlin, Germany

I. Snellen

University of Leiden, Leiden, the Netherlands 
access to liquid water. This constraint led to the definition of the habitable zone (e.g. Hart 1979; Walker et al. 1981; Kasting et al. 1993; Kopparapu et al. 2013), which describes the range of distances from a star where liquid water can exist at the surface of a planet depending on its atmosphere (greenhouse gases like $\mathrm{CO}_{2}, \mathrm{H}_{2} \mathrm{O}$ or He, e.g. Seager 2013).

When searching for a possible habitable exoplanet, the focus often lies on planets with a density indicating a rocky bulk composition. Only few lowmass planets have been detected so far, for which the mass and radius could be measured, and such observations are currently only possible for close-in planets. Detected planets even include small objects in the habitable zone of solar-like stars (e.g. Kepler 425b with 1.6 Earth radii, Jenkins et al. 2015), even though their mean densities are not yet known.

The past measurements show that exoplanets are much more diverse than previously expected, including super-Earths (planets with masses up to 10 Earth masses, Valencia et al. 2007), hot Jupiters (Mayor and Queloz 1995; Marcy and Butler 1996) and mini-Neptunes (Charbonneau et al. 2009; Léger et al. 2009). The latter contain large amounts of water and may migrate inward, possibly into the habitable zone (Kuchner 2003; Léger et al. 2004). These ocean planets have added to the speculation of habitable worlds. Several studies have addressed the possible interior structures and evolution of these lowmass planets in the past years (Sotin et al. 2007; Grasset et al. 2009; Alibert 2014; Noack et al. 2016).

Planets with a small density do not necessarily indicate large amounts of water, but may also contain a thick and broad volatile envelope similar to gas giants. Observations of exoplanets (Rogers 2015) show that there is a threshold between terrestrial planets and planets with an extended atmosphere at a limit of about 1.6 Earth radii (or 6 Earth masses, Marcy et al. 2014).

Direct measurements of water vapour in exoplanet atmospheres have already been obtained for about a dozen objects, mainly hot Jupiters. Such observations are expected to be expanded to planets of significantly lower mass and temperature with upcoming space and ground-based observatories such as the James Webb Space Telescope (JWST) and the next generation of Extremely Large Telescopes.

In this paper we give a summary of recent advances in detection of exoplanets, as well as observation and modelling of water in exoplanets, and discuss possible implications on the habitability of these planets.

\section{Past detection of exoplanets and their bulk compositions}

Up to today, most extrasolar planets are detected by indirect methods. The so far most successful detection methods, the radial velocity (RV) technique and the transit method, fortunately can provide us with the bulk planet parameters orbit, radius, mass, and mean density. Masses can also be derived from astrometric techniques, which will gain significant impact in particular for gas planets when results from ESA's GAIA mission become available. Direct imag- 
ing methods currently assess (young) gas planets and can provide good targets for atmosphere spectroscopy, but concerning bulk parameters (radii, masses) need model assumptions. Here, we focus on RV and transit detections because they provide the largest statistics for planet characterization available today.

\subsection{Techniques to measure planet densities}

Determining planet mean densities requires accurate measures of the planetary radius and mass. The only means we have to directly measure exoplanetary radii is via the transit method. Planetary masses are determined via RV data or by transit timing variations (TTVs) of transiting planets.

The RV technique measures the wobble of a star induced by an orbiting planet around their common centre of mass. The radial velocity component of the stellar motion is measured by high-resolution spectrographs from the induced spectral line shifts via the Doppler-effect. From the periodicity, amplitude, and shape of the signal, the planetary orbit parameters as well as a lower limit of the planetary mass, $m \sin i$ ( $i$ : inclination of the orbital plane), can be derived.

The transit method measures the dimming of the stellar flux when an orbiting planet passes through the line-of-sight towards the star. The dimming is proportional to the projected disk of the planet divided by the disk of the star $\left(k=r_{p}^{2} / r_{s}^{2}\right)$. The periodicity allows deriving the planetary orbit parameters via Kepler's third law. Spectroscopic follow-up observations, e.g., during transit, of the secondary eclipse, and of reflected or emitted light as the planet revolves around its host star, provide access to further planet properties, like atmosphere composition, effective temperature, and albedo (see Section 4).

The two techniques, RV and transit method, complement each other. Planets which can be observed by both methods allow us to remove the $\sin i$ uncertainty in the mass determination and hence provide the true planetary mass, the radius and therefore mean planetary density.

The transit method provides an additional tool to derive planetary masses. Additional planets in a planetary system can induce Transit Timing Variations (TTVs), that means shifts in the occurrence time of an observed transit event. If several transiting planets in a system show TTVs, their masses can be derived with high accuracy. If only one transiting planet is observed and others are indicated only indirectly via their TTVs induced on the observed planet, their masses can still be constraint but with a larger error bar. The derivation of planetary masses for non-transiting planets via TTV is a tricky issue because their orbital parameters (e.g. eccentricity) are often only poorly constraint.

A pre-requisite to the derivation of planet parameters is the good knowledge of the host star. The RV method measures $m \sin i$ of the planet relative to the mass of the host star. The transit signal depends on the ratio of planet to host star radius. Highly accurate determinations of host star parameters are therefore curial for our understanding of the planet properties. Often it is the uncertainty of stellar parameters which limits our knowledge of the orbiting 
planet. Future instruments to investigate exoplanet parameters must therefore be combined with techniques to derive accurate stellar parameters (see Section $6)$.

\subsection{Planet density as constraint to planet interior}

Today about 2000 exoplanets are known (exoplanet.eu). NASA's Kepler mission has published in addition about 7000 planet candidates which are very useful for statistical studies. However, out of the known planets only about 200 are small/low-mass planets with masses $<30 M_{\text {earth }}$ (excluding planet candidates and planets with only mass upper/lower limits), and only about 60 out of these have a measured mean density (exoplanet.eu). The mean uncertainty in their density is about $40 \%$ (where the uncertainty comes from the uncertainty in mass of about $20 \%$ and radius of about $5 \%$ to the power of three). Only few small planets have measured densities with uncertainty $<10 \%$. The number of detected and characterised planets continuously grows, but today's snapshot illustrates the difficulty we have to derive accurate planetary densities with current instrumentation.

Figure 1 displays the density-mass relationship for exoplanets (red and orange dots) and compares it to the Solar System planets and large moons (blue dots). The figure also shows model results for planets with different compositions (after Wagner et al. 2012) to guide our understanding of the planet distribution seen. Planets with Jupiter-like composition fall onto the green-dashed line. This branch of the diagram contains the gas giant planets with composition dominated by hydrogen, similar to Jupiter and Saturn in our Solar System. Their mean density grows with increasing mass. Gas giants falling below the green-dashed line are larger than expected by the standard model and often called "inflated" planets. Planets above the green-dashed line require significantly more heavy elements than Jupiter and may have large rocky cores (e.g. Cabrera et al. 2010; Deleuil et al. 2012). At $\sim 0.3 M_{\text {jupiter }}$ we find a minimum in the mean density versus mass distribution, separating the low-mass planets with significant rocky/icy composition from the gas giants (Hatzes and Rauer 2015; Laughlin and Lissauer 2015). Planet synthesis models (e.g. Mordasini et al. 2012a,b) show that we expect such planet to be numerous.

Low-mass planets in a density range which falls below the light-blue line (ice composition) have significantly extended atmospheres, resulting in large planetary radii and low mean densities. Examples in our Solar System are Neptune and Uranus. We point out that in contrast to the ice planets in the Solar System, up to today basically all small exoplanets with known mean density orbit close to their stars and belong to the class of hot planets. Some of them may have significantly extended atmospheres which make the planet appear very large, in particular in the presence of high-altitude clouds (Lammer et al. 2016). To disentangle from radius and mass observations alone the size of a rocky core from an extended atmosphere is difficult. We take the planet GJ1214b to illustrate the problem. One of the currently best studied 


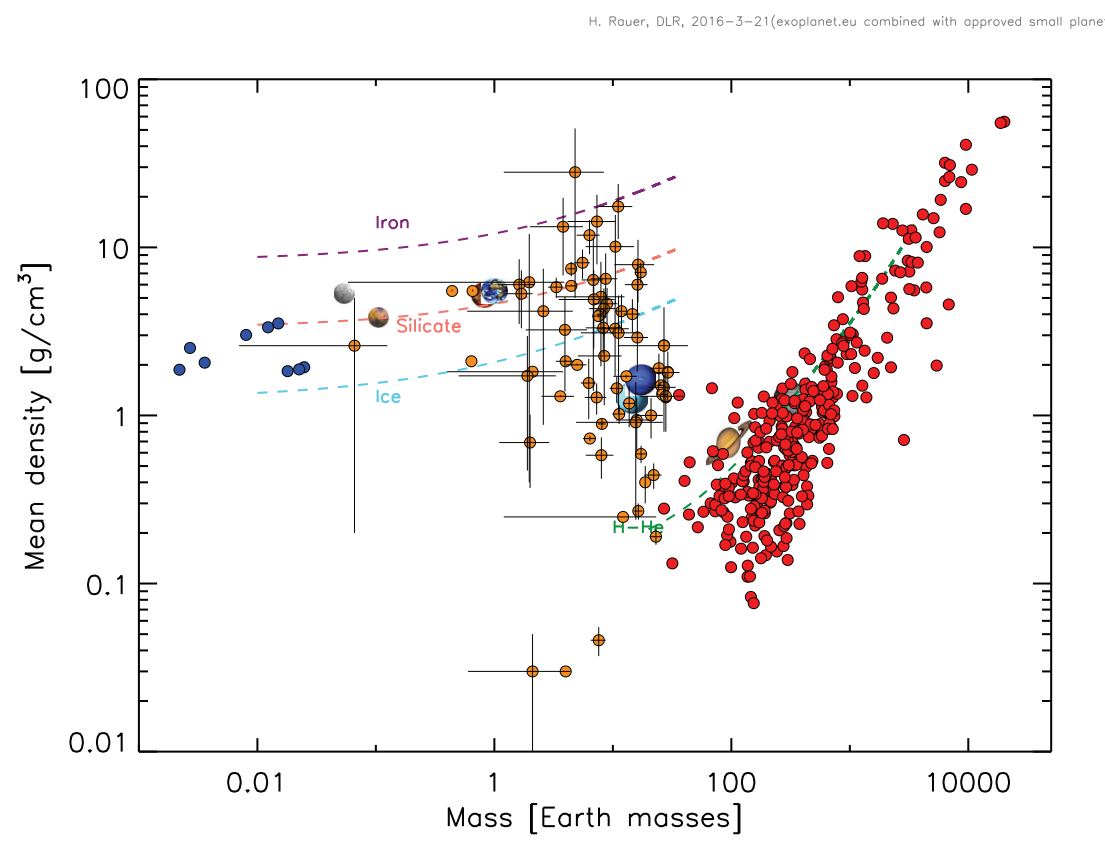

Fig. 1 Mean planet density versus mass. Dashed lines show density-mass relationships for planets with certain composition (green: Jupiter-like, violet: iron, pink: silicate, light blue: water ice). The Solar System planets are indicated by their images and the large Solar System moons by blue dots. Red dots: exoplanet gas giants (no error bars given for clarity), orange dots: low-mass exoplanets.

exoplanets is GJ1214b (Charbonneau et al. 2009). It is a planet with $6.26+/-$ $0.91 M_{\text {earth }}$ and 2.8+/-0.24 $R_{\text {earth }}$ (Anglada-Escudé et al. 2013; Charbonneau et al. 2009), hence belongs to the planets often called "super-Earths". Its mean density is $1.56+/-0.61$. GJ1214b orbits a nearby $(13 \mathrm{pc})$ cool M dwarf star (M4.5) and was subject to intense observations trying to determine the atmospheric composition of the planet. However, no atmosphere absorptions could be detected which was finally attributed to clouds in the upper atmosphere, inhibiting the detection of atmospheric features (Kreidberg et al. 2014b). GJ1214b was also subject to studies to derive its composition and internal structure. In principle three scenarios were studied. All scenarios assume a planet with an iron core and a silicate mantle layer, but they differ in the assumed atmosphere composition. Atmospheres could be dominated by hydrogen on top of the silicate mantle, or on top of a water ice layer. A third atmosphere scenario assumes a water steam atmosphere, resulting from e.g. evaporation of a thick ice layer when the planet migrated towards the star during its early evolution. It was shown that all three scenarios are able to meet the measured planetary radius and mass (Rogers and Seager 2010), but 
require different amounts of hydrogen and water. This was confirmed in similar studies, showing that it needs $<10 \%$ hydrogen above a rocky core to explain the measured radius and mass, or alternatively large amounts of water in the atmosphere (Rogers and Seager 2010; Nettelmann et al. 2011; Valencia et al. 2013). GJ1214b illustrates that from radius and mass measurements alone it is not possible to disentangle this ambiguity. Detection of atmospheric compositions in planets could help. However, it will also require a better understanding of planet formation and evolution scenarios to build arguments to constrain probable planet compositions in this planet parameter range.

Mean densities of rocky terrestrial planets similar to Earth are dominated by their iron cores and silicate mantles. The atmosphere contributes only little. These planets fall between the violet and pink lines in Figure 1. We can see that Mars, Venus and Earth line-up on the silicate composition line. The figure also shows that there seems to be an upper mass limit for such silicate-dominated planets. The highest-mass silicate planets observed so far have masses of 10$20 M_{\text {earth }}$ (Rauer et al. 2014). This is in agreement with the core-accretion model of planet formation which expects larger planets, beyond the critical core mass, to accrete hydrogen and quickly grow in size (and hence decrease in mean density, falling below the light-blue line or moving to the right part of the diagram of gas giants). Also Mercury-like planets which require large iron cores to explain their mean density are found. However, error bars in this lowmass part of the diagram are large, and a higher accuracy of measured mean densities is urgently needed to at least minimise the ambiguities from observations. However, also planets in this low-mass parameter range are subject to compositional degeneracy (see Section 3).

The examples above have shown that inferring the water content of planets from their bulk properties, radius, mass, and density, is a challenging task due to compositional degeneracies between core, mantle (silicate and/or ice) and atmosphere contributions. Nevertheless, statistical methods to disentangle terrestrial planet composition from radius and mass data, taking into account measurement and model uncertainties, are currently investigated (Dorn et al. 2015). These studies show that constraints may become available with an increasing data base of measured planet (radius, mass, mean density, atmosphere) and host star (composition) parameters. While the determination of a precise composition and water content of a particular, individual exoplanet will likely remain a challenge, improved data quality and especially large sets of observations will provide a new parameter input into the analysis of statistical properties of terrestrial planet composition in future.

\section{Interior structures from bulk compositions}

When detecting small-massive exoplanets, a rough characterisation of the bulk composition can be obtained when comparing the observed mass and radius to predicted relationships for different planets types - typically ranging from pure water spheres over silicate shells to iron-rich bodies (e.g. Valencia et al. 2006; 
Wagner et al. 2012; Dressing et al. 2015; Noack et al. 2016). An exoplanet that would lie on the mass-radius curve of an Earth-like structure would therefore be assumed to be rocky and similar to Earth. However, interior structure of planets and composition is never unambiguous.
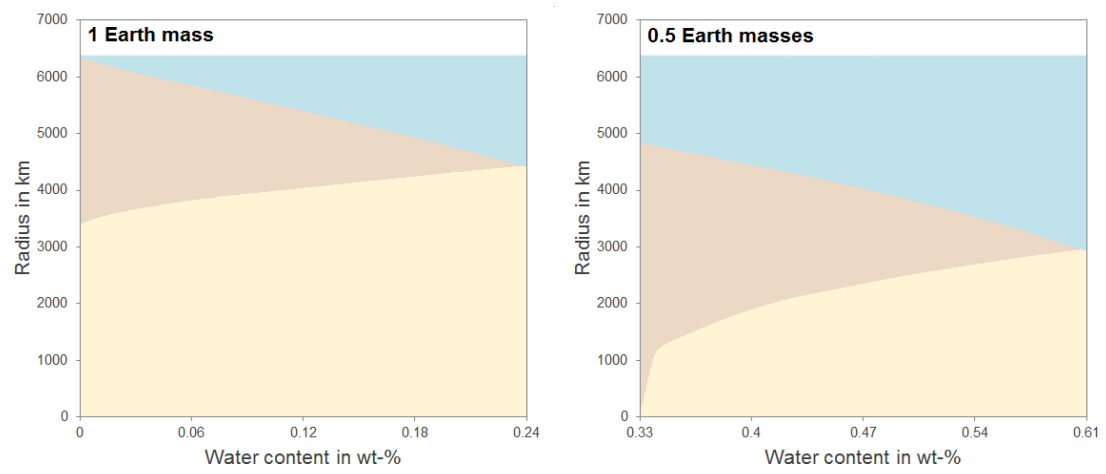

Fig. 2 Possible interior structure variations for an Earth-size planet of 1 (left) or 0.5 (right) Earth masses composed of an iron core (yellow area), a silicate mantle (brown) and water/ice (blue).

Figure 2a shows a simplified example for different interior structures that are possible for an Earth-size planet with a mass of exactly Earth's mass (neglecting measurement inaccuracies here). The blue areas denote a pure water layer (with a surface temperature of $300 \mathrm{~K}$ and consisting of liquid water and possibly high-pressure ice), brown areas are the silicate mantle (containing only the minerals $\mathrm{Mg}_{2} \mathrm{SiO}_{4}, \mathrm{MgSiO}_{3}$ and $\mathrm{MgO}$ for simplicity) and yellow indicates the iron core (solid in this example). Earth's surface water content is about 0.023 wt-\% (Schubert et al. 2001). However, if Earth would have a larger water content (here up to 24 wt-\% or appr. 1000 Earth oceans), larger amounts of iron could compensate the effect of the less-dense water on the mass of the planet and yield the same observed mass and radius as Earth. Note that a pure water-iron planet lacking any silicate minerals is unlikely, but variations in the $\mathrm{Mg}-\mathrm{Fe}-\mathrm{Si}$ ratios have already been observed for several stars - which may indicate related variations in planet compositions and can help to constrain possible interior structures (e.g. Grasset et al. 2009; Santos et al. 2015; Dorn et al. 2015; Unterborn et al. 2016).

The right plot in Figure 2 on the other hand shows a planet of one Earth size but only half the mass of Earth. As long as an extended atmosphere, which would yield a larger observed than actual planet radius (e.g. Lissauer et al. 2011), can be excluded (for example for low-massive planets up to Earth's mass Lammer et al. 2014), such a small bulk density can only be explained for substantial amounts of water. For such planets a rough characterisation based on their mass-radius relationship is possible. For planets discovered with Earth- 
like densities, large amounts of water cannot be excluded, and the detected exoplanet may have either a water-rich or a rocky composition.
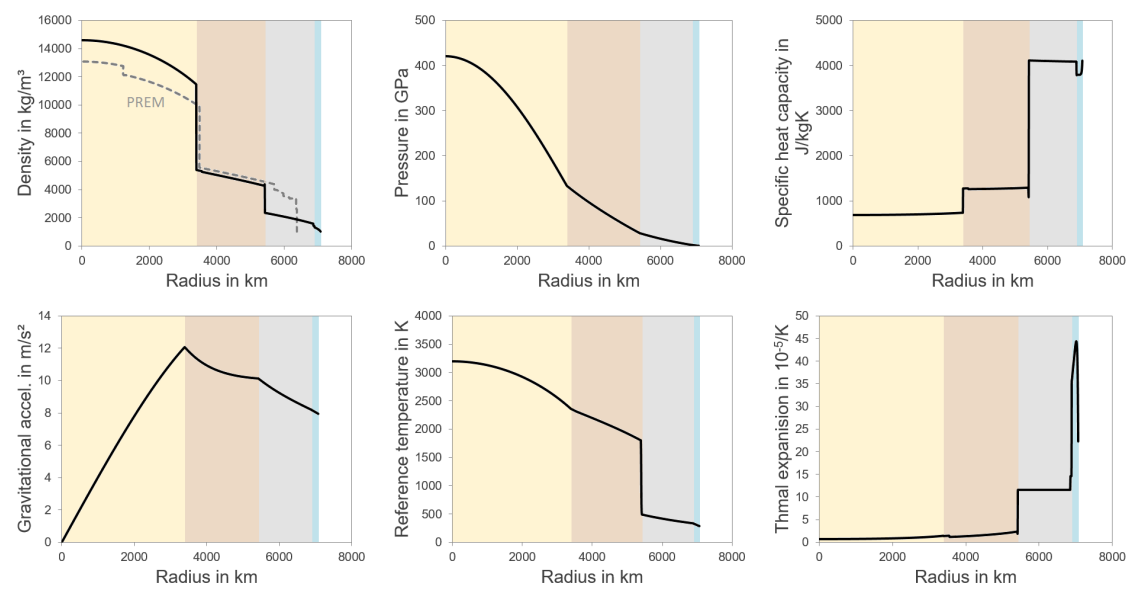

Fig. 3 Radial profiles of a water-rich planet for density, pressure, and different thermodynamic properties for a one-Earth-mass planet composed of $35 \mathrm{wt}-\%$ iron, $25 \mathrm{wt}-\%$ water and 40 wt- $\%$ silicates from Fig. 2. The yellow colour denotes the iron core, brown the silicate mantle, grey the high-pressure ice and blue the liquid water layer at the surface. Possible melting of the ice layer from below is neglected here for simplicity. The grey dashed line shows the density of the preliminary reference Earth model (PREM, Dziewonski and Anderson 1981).

In Figure 3 example profiles of a water-rich planet throughout the interior are plotted for the density, pressure, specific heat capacity, gravitational acceleration, adiabatic reference temperature and thermal expansion coefficient after Noack et al. (2016). This model planet of one Earth mass contains $35 \mathrm{wt}-\%$ iron and $25 \mathrm{wt}-\%$ water. Distinct variations of several thermodynamic properties for the different layers (water - mantle - core) can be observed for the density and the thermal properties. For comparison, the density profile of the Earth following the preliminary reference Earth model (PREM) by Dziewonski and Anderson (1981) is given. The main differences between the density profiles can be observed in the upper first $1000 \mathrm{~km}$, where the large water content of the modelled planet leads to smaller densities in the high-pressure ice layer than in the silicate upper mantle of Earth. The density of Earth's core is reduced with respect to the model planet due to existence of lighter elements in Earth's core, which were not considered in the model planet. While mass and radius of rocky planets can be observed from space, the interior structure as well as information on thermodynamic properties of the different layers in the interior cannot be observed. Models of possible planet compositions and differentiation processes can help to better understand the thermal evolution of the interior of rocky planets, which influences the subaqueous surface, for example via volcanism and plate tectonics (Noack et al. 2016, 2017). 


\section{Characterisation of exoplanet atmospheres and observing water}

Decades of Solar System exploration have revealed an immense complexity and diversity of planet atmospheres. Gas giants' atmospheres are molecular hydrogen dominated and exhibit strong zonal flows with thick clouds and storms. The rocky planets harbour secondary atmospheres, with a super-rotating $\mathrm{CO}_{2}$ based atmosphere with opaque sulphuric acid clouds in the case of Venus, a $\mathrm{N}_{2}$ based partially clear atmosphere with a significant biotic oxygen content in the case of the Earth, and a tenuous $\mathrm{CO}_{2}$ based atmosphere with yet unexplained trace amounts of methane in the case of Mars (Webster et al. 2015). Arguably even more mysterious is the thick $\mathrm{N}_{2}$-based atmosphere of Saturn's moon Titan, with opaque methane/ethane clouds.

Scientifically, the daunting task is to understand this wide variety in atmospheres in the frame work of planet formation and evolution. Since the atmospheres of the Solar System planets are so different from each other, it indicates that it will require detailed investigations of extrasolar planets to even begin to recognise patterns, relations, and/or families between different types of planets. Planet types such as hot Jupiters, super-Earths and miniNeptunes do not exist in our Solar System.

On the other hand, studying the atmospheres of extrasolar planets will always be significantly limited compared to what we can do for Solar System planets. The latter we can send probes to that dig and drill to understand their geology, or that perform in situ atmospheric measurements. Artificial satellites can accurately measure a planet's gravity field, and landers can collect seismic data, or in the future even collect samples that could be returned to Earth. All these important and basic observables will never be available for exoplanet studies.

Fortunately, a wide variety of information can be obtained from remote sensing alone. Moreover, the composition, thermal structure and dynamics of a planet atmosphere can be studied using a wide range of observational techniques.

\subsection{Techniques to measure exoplanet atmospheres}

In the first two decades since the first discoveries more than 1000 exoplanets have been found. Virtually all of these planets have been discovered without identifying even one single photon from the planet itself. Unfortunately, this can no longer be the case for atmospheric studies, and such observations are about separating the planet signal from that of the star, with the latter often many orders of magnitude brighter.

Practically all aspects of astronomical observations, which measure the radiation (and polarisation) as function of time, direction, and wavelength, can be used to filter out the light from the planet: 


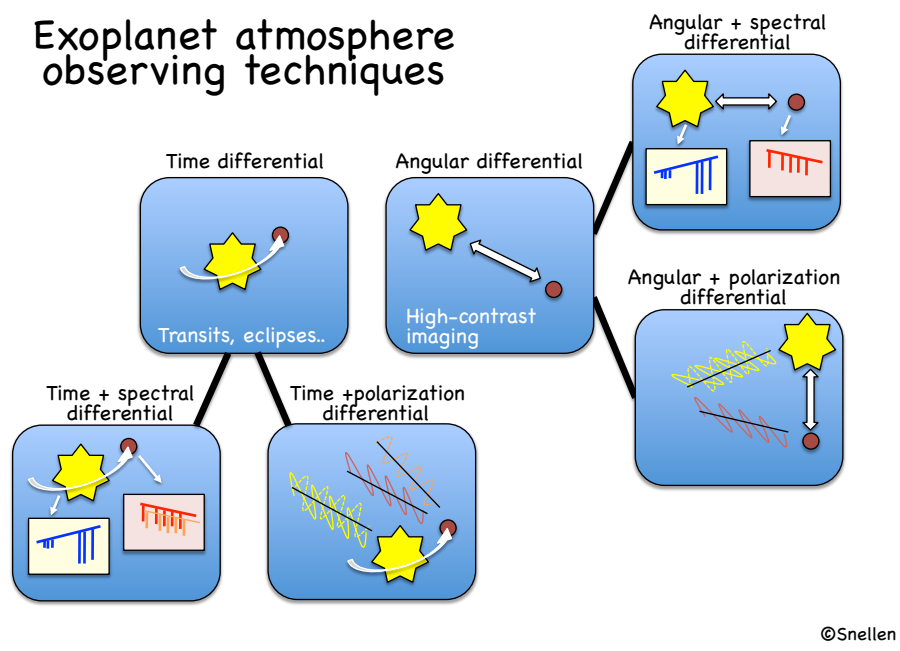

Fig. 4 Exoplanet atmosphere observations are all about separating the planet light from that of the star. This can be done using time differential measurements (using transits, eclipses, or phase curves), angular differential techniques using high-contrast imaging, and either of these combined with high-dispersion spectroscopy or polarisation observations.

1: Time domain - transits and eclipses: The way a planet is observed can be a dramatic function of time. This is particularly the case for transiting systems. Not only does the planet at given times block off part of the stellar surface, but a small fraction of star light filters through the planet atmosphere leaving a spectral imprint of absorption and scattering processes. By observing the system as function of time, the planet's atmospheric transmission spectrum can be isolated from the stellar spectrum (Charbonneau et al. 2002).

This works best when the observability of the planet changes on short time scales relative to the duration of the observation, such as in the case of transits, or eclipses - the moment the planet vanishes behind the star and the missing radiation is a measure of the planet's thermal emission spectrum and/or reflectance (Charbonneau et al. 2005; Deming et al. 2005). Along a planet orbit we also see the planet at different viewing angles, showing varying fractions of its day and night side. The time scales of such phase curve measurements are longer with the changes in planet signal less abrupt making this significantly more difficult to observe (Knutson et al. 2007).

The planet transmission spectra probe the atmosphere at the day/night terminator region. The atmospheric signal largely depends on the scale height and the abundance of spectroscopically active species and scattering or absorption by aerosols. A secondary eclipse spectrum or phase curve measurement 
probes a combination of star light reflected off a planet's atmosphere and its intrinsic thermal spectrum - the latter largely depending on the atmospheric temperature structure and abundances of molecular species on the planet's dayside. Note that this type of exoplanet atmospheric measurements, which depend on time differential filtering, is mainly constrained to planets in closein orbits, since both the transit probability and transit frequency scale down with orbital distance, but also because these planets are very warm due to the close proximity of their host star.

2: Spatial domain - high contrast imaging: The small angular separation between star and planet can also be used to isolate the planet signal, called direct or high contrast imaging (HCI). Since the theoretical angular resolution is proportional to the telescope diameter, best results so far are being reached with the large ground-based telescopes making use of adaptive optics systems aimed to cancel out the negative effects of Earth-atmospheric turbulence (seeing), coronography to optimally darken regions directly around the star, and utilising smart analysis algorithms that further remove telescope-induced systematics. The hot Jupiters that are being probed with transit measurements are inaccessible with direct imaging since they are too close to the host star. Mature planets that are located at such large orbital distance that they could be angularly separated from their host star are extremely faint, both in thermal as in reflected light, with planet/star contrasts of $10^{-8}$ or lower. This means that they are also beyond reach of current instrumentation. However, very young systems with planets at tens or more astronomical units can be observed (Marois et al. 2008; Lagrange et al. 2010). Although these planets receive only little energy form their host stars, they are still warm from their formation resulting in a much more favourable planet-to-star contrast. In particular young A-stars, which are 2-3 times more massive than the Sun, sometimes exhibit gas giant planets at large orbital distances of which a dozen or so are now known. HCI immediately allows the production of a planet thermal spectrum by combining measurements at different wavelengths (Currie et al. 2010).

Both the filtering in the time-domain as in the spatial domain can be used in combination with filtering on other aspects of astronomical observations. E.g. high-dispersion spectroscopy (HDS) can be used in conjunction with transit or phase-curve observations. At $\lambda / \Delta \lambda \approx 100,000$, a planet molecular band (such as from $\mathrm{CO}$ or $\mathrm{H}_{2} \mathrm{O}$ ) resolves into tens or hundreds of individual lines. Since the orbital motion of hot Jupiters is large (up to $150 \mathrm{~km} / \mathrm{s}$ ) the radial component of the orbital velocity of the planet can change by tens of $\mathrm{km} / \mathrm{s}$ during an observation - adding a time differential component to the observation. This allows the planet also to be separated in wavelength-space by filtering out all stationary components in the observed spectra (including the spectral features from the star and Earth atmosphere). The signals from the individual molecular lines can be subsequently combined using cross-correlation techniques (Cameron et al. 1999; Snellen et al. 2010). 
High-dispersion spectroscopy can also be combined with high contrast imaging (HDS+HCI). While the planet-star contrast obtained with HCI may not be sufficient to detect the planet, the remaining starlight at the planet position may have a significantly different spectrum than that of the planet. The stellar spectrum is well sampled and can be filtered out, leaving the planet signal only at the planet position of which the signal of individual lines can be combined again by using cross-correlation techniques (Sparks and Ford 2002; Riaud and Schneider 2007; Snellen et al. 2015). In this way the spin-rotation of the directly imaged planet $\beta$ Pictoris b was determined for the first time (Snellen et al. 2014).

Similar techniques can also be used in conjunction with polarisation. E.g. it is expected that reflected light from a planet is partially polarised, while that of the star is not. Hence, by isolating the polarising flux, the contrast between the planet and star can be further enhanced. The Zimpol instrument, recently installed on the Very Large Telescope, has been designed to pursue this (Milli et al. 2013).

\subsection{Observations of water in exoplanet atmospheres}

The earliest report of the detection of water vapour in the atmosphere of an exoplanet is from Tinetti et al. (2007) using the Spitzer Space Telescope, who attributed the observed variation in transit depth between three infrared wavelengths as a signature of water absorption in the hot Jupiter HD189733. The same planet was also shown to have water using observations with the NICMOS near-infrared spectrograph at the Hubble Space Telescope (HST, Swain et al. 2008), along with a heavily disputed claim of methane absorption. Charbonneau et al. (2008) and Grillmair et al. (2008) showed that the broadband Spitzer spectrum of the thermal emission spectrum (obtained through secondary eclipse measurements) is also consistent with absorption by water vapour. With the installation of the WFC3 camera on the HST and the utilisation of innovative observational techniques that minimise its systematic effects (drift scanning), the 1.4-1.7 micron water absorption band has been detected for about a dozen hot Jupiters in their transmission spectrum (Deming et al. 2013; Mandell et al. 2013; McCullough et al. 2014; Wakeford et al. 2013), and in the thermal phase curve of WASP-43b (Kreidberg et al. 2014a).

While the strength of the water absorption feature shows planet to planet variations by up to an order of magnitude, Sing et al. (2016) show that this is anti-correlated with the strength of the UV-optical blueward slope of these planets' transmission spectra, generally attributed to Rayleigh scattering of haze particles in the atmospheres. Therefore it seems that it is not a variation in water abundance that is causing the variation in absorption strength, but the presence or not of hazes that partly block the 1.4-1.7 micron water feature.

Pushing this technique to smaller and cooler planets has been a challenge. For several planets (e.g. Kreidberg et al. 2014b; Knutson et al. 2014) the transmission spectrum seems to be dominated by clouds, basically hiding the water 
absorption signature (note that in such hot planets these are not water-clouds). Fortunately, at least one Neptune size planet at $<1000 \mathrm{~K}$ has a spectrum that is not dominated by clouds (Fraine et al. 2014). However, it is clear that clouds may be an important obstacle when searching for absorption features in transmission spectra of cooler planets.

Ground-based HDS of hot Jupiters has also resulted in several water detections. Water absorption was detected in the dayside thermal spectrum of hot Jupiter HD189733 using CRIRES at the Very Large Telescope (Birkby et al. 2013), and in that of tau Bootis using NIRSPEC at Keck (Lockwood et al. 2014) - both at 3-3.5 $\mu \mathrm{m}$. Water absorption was also observed at shorter wavelengths (e.g. Brogi et al. 2014; Schwarz et al. 2015).

HCI has also revealed water absorption in young massive gas giants at large orbital distances. Both planets b and c that orbit the young A-giant HR8799 have been found to exhibit a clear water absorption signature, together with carbon monoxide and methane, and carbon monoxide respectively (Barman et al. 2015; Konopacky et al. 2013). As expected, water vapour is also common in the atmospheres of young, self-luminous gas giant planets.

\section{Planetary evolution and habitability}

The basic definition of habitability, based on life as we know it, is the existence of liquid water as prerequisite - even though other factors like the existence of building blocks of life, energy sources, and nutrients are necessary, as well. Looking at the planets and moons in the Solar System, and at the data of the so far discovered exoplanets, suggests three different groups of bodies that could host life:

- terrestrial planets with shallow, regional water oceans,

- icy bodies with subsurface niches (either subsurface access to water as suggested for Mars or subsurface oceans like for Europa and Enceladus), or

- water-worlds with water-steam atmospheres and liquid water at the surface

In the following, we shortly discuss studies that investigated the habitability potential of terrestrial and icy bodies, followed by a section on water-worlds.

\subsection{Water on terrestrial exoplanets}

The holy grail in exoplanet research is to discover a potential second Earth. While based on the geophysical definition an Earth-like planet (or rocky planet) would solely require a density indicating a composition of mainly silicate minerals and metals, the "second Earth" is typically expected to have surface oceans like on Earth - and therefore needs to be in the habitable zone. Just the right mass and radius would not justify to call the detected planet a "second Earth". Some scientists would in addition request the existence of a magnetic 
field as well as plate tectonics, in other words, a planet that might be able to host complex, Earth-like life (either in its original or present-day form).

The existence of small amounts of water on terrestrial planets cannot be detected from the mass-radius relationship (see Section 2, 3). On the other hand, when searching for complex life like it evolved on Earth, the existence of surface land (volcanic islands or continents) may be crucial, and only shallow ocean layers may be considered as habitable (Maruyama et al. 2013).

The detection of water and continents at the surface of a terrestrial exoplanet could be possible at some point. Water in the atmosphere can be detected from its spectrum, see Section 4. Observation of surface coverage of oceans and land might be possible due to interpretation of light curves and albedo measurements (Montanes-Rodriguez et al. 2006; Fujii et al. 2010) for non-transiting planets.

Another question concerning water at the surface of terrestrial planets is its survivability. During the long-term evolution of Sun-like stars (F- or Gtype stars), the solar flux strongly increases with time. The standard model for the adaption of the atmosphere to changing solar radiation, as used in the calculation of habitable zone boundaries (e.g. Hart 1979; Kasting et al. 1993; Kopparapu et al. 2013), includes the formation of carbonates to extract $\mathrm{CO}_{2}$ from the atmosphere to compensate for the increasing solar flux. This process may already need the existence of plate tectonics to subduct carbonates produced at the continental shelves, which may be limited by several factors including for example the planet mass (still under debate, e.g. Valencia et al. 2007; van Heck and Tackley 2011; Noack and Breuer 2014), interior heat (Stein et al. 2013; Stamenković and Breuer 2014) or composition (Noack et al. 2014). Inefficient atmosphere adaptation to increasing solar flux would lead to a runaway greenhouse effect and loss of surface water. On the other hand, limitations to the maximal potential amount of greenhouse gases in the atmosphere could have severe consequences for the existence of liquid surface water at the outer boundary of the habitable zone (Noack et al. 2014).

Another interesting contribution of plate tectonics is the recycling of water into the mantle and its replenishing at volcanic regions (Parai and Mukhopadhyay 2012; Höning et al. 2014; Foley 2015), which may be able to lead to stable, shallow ocean layers - even for slightly larger amounts of water than on Earth leading to an enrichment of water in the mantle instead of deeper surface ocean layers for small-massive planets (Schaefer and Sasselov 2015).

\subsection{Water in icy bodies}

While early Mars had periods of liquid water flowing locally at the surface (McKay and Davis 1991; Halevy and Head III 2014; Helbert et al. 2015), present-day Mars can rather be considered as an icy world (apart from few regions at the equator where temperatures rise above zero degrees during the day). In the subsurface of Mars, access to liquid water may be possible, and if life did exist during the earlier stages of the evolution, it may have penetrated 
into the ground, where it is also shielded from surface radiation (Boston et al. 1992; Gold 1992).

The more traditional view of icy bodies considers the water-rich moons in the outer Solar System covered by an ice layer. These moons formed outside of the snowline. Icy moons are typically considered to be habitable, if a subsurface ocean exists, which is in contact with the silicate mantle. Hydrothermal activity or serpentinisation processes within cracks of the rocky core (e.g. Vance et al. 2016) are needed as energy source, and access to nutrients is furthermore essential for long-time habitability of these subsurface oceans. Subsurface oceans are believed to exist within Ganymede (Kivelson et al. 2002; Saur et al. 2015; Vance et al. 2014), Titan (Iess et al. 2012; Grindrod et al. 2008) and Callisto (Zimmer et al. 2000; Khurana et al. 1998), but at depth highpressure ice forms, which hinders the direct access of the ocean to the rocky core (even though for Ganymede an upper and lower water layer have been suggested, Vance et al. (2014)). The moons experience negligible radioactive heating from within and only limited tidal heating, such that melting of the entire high-pressure ice layer from beneath is not expected. Therefore, in the Solar System, typically only Europa and Enceladus are considered as habitable icy moons, where water-silicate contact is possible (e.g. Roth et al. 2014; Khurana et al. 1998; Thomas et al. 2016; Van Hoolst et al. 2016).

\subsection{Habitability of ocean planets}

The detection of low-dense, massive exoplanets (see also Section 2) has started the discussion about the habitability of large water-rich planets, often called ocean planets or mini-/sub-Neptunes (Léger et al. 2004; Grasset et al. 2009; Sotin et al. 2007; Alibert 2014; Luger et al. 2015; Lammer et al. 2009; Noack et al. 2016). These planets need to contain large amounts of water and/or extended atmospheres to explain their low density (see Section 3).

Of interest have been especially water worlds in the habitable zone, where the hydrogen-helium atmospheres allow for liquid water at the surface of these planets. Sub-Neptunes outside the snowline could be potentially habitable, as well, although observations will be extremely challenging due to the enormous star/planet contrast ratio (if they can be observed, at all, and are not scattered out of their star system, Stevenson 1999). Furthermore, biosignatures in the atmosphere indicating life would be enhanced on planets without a surface ice crust, where life could extend towards the surface.

Alibert (2014) modelled the maximal possible habitable planet radius for water worlds of different masses considering different mantle-core composition. For large water amounts, high-pressure ice can form, which is typically considered to rule out habitability of the ocean, as the ocean is not in contact with the silicate part of the planet. Similar studies have investigated the maximal liquid ocean depth (e.g. Sotin et al. 2007), also under the aspect of different surface temperatures (Noack et al. 2016). 
The recent study by Noack et al. (2016) looked closer at water-rich planets to evaluate the decrease in habitability potential with increasing water amount. The study found that, depending on several factors such as initial energy budget, amount of radioactive heat sources, or composition, high-pressure ice can be molten from beneath due to the high heat flow from silicate mantle into the ice layer. In some cases, only an episodic second, lower ocean layer appears above the silicate mantle. Such a lower ocean layer (episodic or not), would classify as a habitable niche inside water-rich planets.
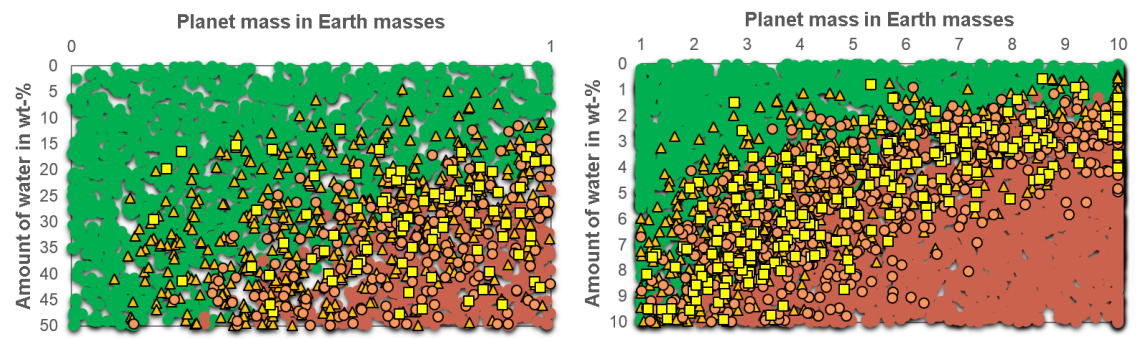

Fig. 5 Possible habitability evaluation for planets of variable masses, compositions and interior parameters. The colours refer to liquid water layers (green), lower ocean layers (yellow), episodic lower ocean layers (orange) and high-pressure ice layers that are not molten at the bottom (red). Based on Noack et al. (2016).

To understand how the possible habitability of a deep water layer (possibly including high-pressure ice and a second, lower ocean above the silicate mantle) depends on the amount of water and mass of a planet, we model the thermal evolution of 7500 model planets over several billion years assuming arbitrary values for composition, initial parameters (as for example the amount of radioactive heat sources or initial temperature profile), crustal production parameters and different silicate lithosphere regimes (stagnant lid or plate tectonics) following a Monte Carlo approach as in Noack et al. (2016).

Fig. 5 shows the habitability potential of the model planets separated into a low-mass range (left plot) and super-Earth mass range (right plot). For each planet, we determine if high-pressure ice forms during the evolution of the planet, and if the heat from the interior would be sufficient to melt the ice layer from beneath. The colours in Fig. 5 refer to the possible habitability of the water-rich planet. The green region is the region where no high-pressure ice forms, whereas in the yellow and orange cases a lower ocean forms (only episodically for planets with orange dots). For model planets with a red dot, a high-pressure ice layer forms, which extends to the surface of the silicate shell. These planets are likely uninhabitable Noack et al. (for more information on the habitability classification we refer the reader to 2016). When taking into account planets with a second, lower ocean in the classification of possibly habitable water-rich planets, the range of water amounts that still allow for habitable worlds is extended to much larger values than compared to the classical restriction of having no high-pressure ice forming in the wa- 
ter layer. Note, however, that the $\mathrm{CO}_{2}$ feedback cycling between interior and atmosphere would be hindered, which may have additional consequences for the atmosphere stability and habitability (Kitzmann et al. 2015). For small massive planets (from Mars' to Earth' mass), immense water contents up to several tens of wt-\% could still allow for the origin and evolution of life as we know it, whereas for massive super-Earths, even small amounts of water in the range of few wt-\% would make the planets uninhabitable.

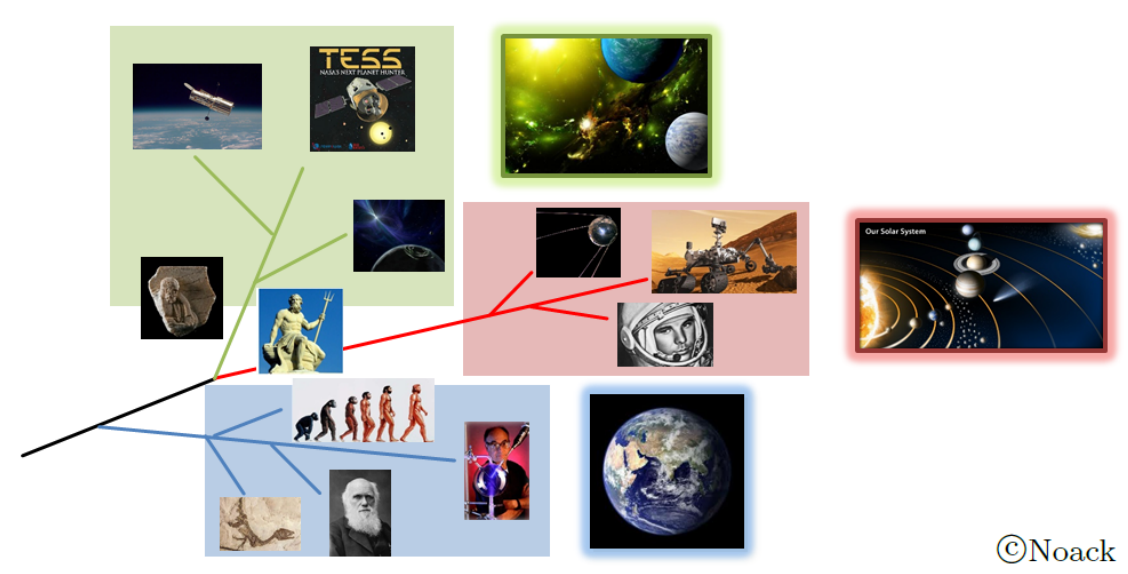

Fig. 6 Tree of life awareness. The different-coloured branches indicate different evolution steps of the search for life, from the first thoughts on the history of life (blue branch) to search for life on other planets or moons in the solar system (red branch) and in the universe (green branch).

Figure 6 shows a different tree of life than the well-known biological tree of life (Darwin 1859; Hinchliff et al. 2015). The figure summarises the evolution of the search for life, starting with the first thoughts on the history of life, which is indicated in the lower, blue region. Archaeological findings and the fundamental works of Charles Darwin and others led to the discovery of life's origin on Earth, including the ground-breaking Miller-Urey experiment creating amino acids under early-Earth conditions in the laboratory and thus creating organic compounds, that are essential for life as we know it.

A different branch of life awareness, considering life on other planets, goes back to Greek philosophers such as Democritus around 400 BC, who claimed that the universe must contain more than one planet sustaining life. The red area in Fig. 6 refers to planets and moons in the Solar System. Mars, the moon and Venus were believed to be inhabited (see also review in Noack and Breuer 
2013), until the area of space missions revolutionised the idea of life on other planets.

In the last two decades, after not having found life on another body in the Solar System, and after the first exoplanets were discovered in the early 1990s (Wolszczan and Frail 1992; Mayor and Queloz 1995), the universe is in the focus of the search for life (green region). The hunt for finding possible habitable exoplanets started a decade ago and continues with numerous upcoming detection and characterisation missions such as TESS (Ricker et al. 2015), CHEOPS (Broeg et al. 2013), JWST (Sabelhaus and Decker 2004) and PLATO (Rauer et al. 2014), see also Section 6. One goal of these missions is to find a second habitable world - a planet containing liquid water that life can access, and where it can flourish.

\section{Outlook missions}

6.1 Bulk planet characterisation: Radii, Masses, Density and Ages

Figure 7 provides an overview of past and future missions relevant for exoplanet studies. The first dedicated space mission for exoplanets CoRoT (CNES, Baglin et al. 2006) detected 32 exoplanets so far, all with RV follow-up observations and hence measured radii and masses. CoRoT provided us with the first secured terrestrial planet, CoRoT-7b (Léger et al. 2009). NASA's Kepler mission (Koch et al. 2010) detected thousands of planet candidates, including $\sim 80$ planets for which RV follow-up was possible and hence mean planet densities could be derived, including the small terrestrial planet Kepler-10b (Batalha et al. 2011). Both missions stopped nominal operation in 2012. However, the Kepler satellite continuous to take data, now as K2 mission (Howell et al. 2014). K2 observes several target fields near the ecliptic plane for durations of up to about 80 days each.

CoRoT, Kepler and K2 benefited from the availability of space observatories such as HST and the IR telescope Spitzer, since planets around sufficiently bright stars could be followed up with space-based spectroscopy to study their atmospheres (see below).

NASA's exoplanet transit survey TESS (Ricker et al. 2014) will provide the first all-sky survey to search for transiting planets. Most target fields will be observed for periods around $\sim 1$ month, but a small continuous viewing zone allows for observations of up to one year. TESS is planned for launch in 2018 and will hence be able to provide targets for JWST. TESS targets will be bright and radii, masses, and mean densities will be known for most of its detections.

In Europe, ESA will launch its first small mission CHEOPS (Broeg et al. 2013) in 2018. CHEOPS is a follow-up mission and will determine accurate radii for planets detected previously by the $\mathrm{RV}$ method, but also refine radii of TESS targets and ground-based transit detections. In 2025, ESA is planning to launch its M3 mission PLATO (Rauer et al. 2014). PLATO's main target range 


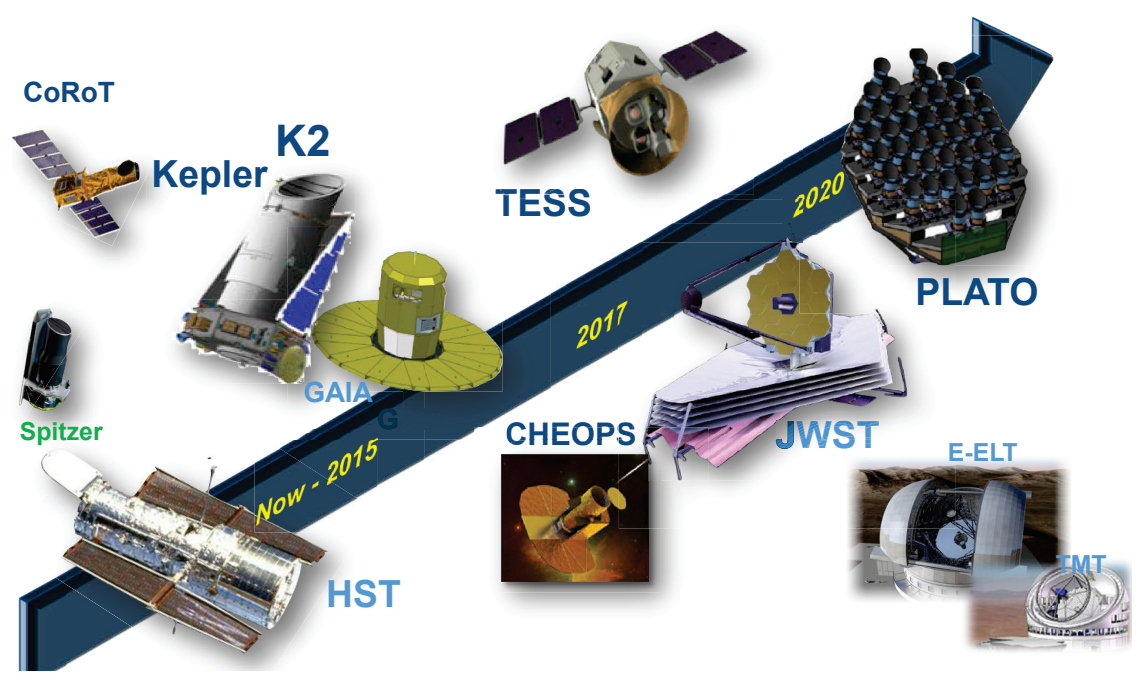

Fig. 7 Overview of past and future missions relevant for exoplanet characterization.

is small planets, including characterised planets in the habitable zone. PLATO will provide not only accurate radii and masses (from RV follow-up), but also accurate stellar parameters from asteroseismology. This technique was first applied to space photometric data by CoRoT and then successfully applied to the brightest Kepler targets. PLATO will now perform asteroseismology of host stars on a large scale to obtain not only accurate radii and masses, but also well-known ages of a large number of planetary systems. PLATO targets will be observable in a JWST extended mission phase and come in time for the 30m-class ground-based telescopes E-ELT (ESO) (Ramsay et al. 2014) and TMT (Skidmore et al. 2015).

In the next two decades we will see a wealth of data of planets characterised for their radius, mass and mean density, allowing for statistical studies of characterised planet samples. Many of these will be suitable for spectroscopic follow-up to measure their atmospheric composition.

6.2 Atmospheric observations: technological and observational developments

Current state of affairs is that for gas giants that are hot (>1000 K), either through irradiation of their host star or because they are young and still contracting, water absorption features can be readily observed. First attempts to push this to smaller planets and those with lower temperatures were so far only 
partially successful, hampered by the occurrence of clouds, which in particular in the case of transmission spectroscopy can mask absorption features.

The JWST, which will be launched in 2018, will constitute an enormous enhancement over the HST. It's collecting area is a factor 7 higher, and importantly the JWST will be flown to L2, meaning that in stark contrast to the HST it will be able to observe most targets for long periods in time (compared to the low-Earth 95-minute orbit of HST during which the Earth eclipses a target for significant periods). Stability is key for transmission, eclipse and phase curve observations and the JWST will provide this. In the case of clear atmospheres, the JWST is expected to be able to reach detection of water vapour for planets significantly lower in mass and lower in temperature than the HST can provide, possibly in the super-Earth or even Earth-size regime. However, clouds may spoil the party. In that case, observations at mid-infrared wavelength with MIRI may provide a solution - probing wavelength long enough such that observations through the clouds may be made.

Ground-based high-dispersion spectroscopy may be another solution to deal with cloudy atmospheres. The high-dispersion means that observations probe the atmospheres at significantly higher altitudes, being much more sensitive to water absorption features above any (non-water) cloud layers. New or upgraded spectrographs at existing 4-10 m class telescopes will significantly push this method forward, with as ultimate instruments the upcoming extremely large telescopes such as the E-ELT, both for high-dispersion and highcontrast imaging, of which the observing speed scales with the telescope diameter to the 4th power. Planned high-dispersion spectrographs such as METIS in the mid-infrared and HIRES in the optical near-infrared will revolutionise this field and may be able to characterise the atmospheres of Earth-like planets in the habitable zones of nearby stars.

\section{Discussion and summary}

The detection of water (as well as its amount) is ambiguous when using mass and radius measurements alone. On the one hand, the current mean uncertainty of measured mean densities for small planets is about $40 \%$. On the other hand, even if the exact mass and radius of a planet would be known, its composition cannot be derived unambiguously from the data alone. Together with information on the star's composition and the planet's surface temperature, statistical methods can suggest likely compositions (Dorn et al. 2015; Santos et al. 2015). But even for the planets in the Solar System, this information gives only a rough understanding of the interior structure of the rocky planets, and seismic measurements are needed for an improved understanding of the planets' compositions. This is not possible for exoplanets and will always limit our understanding of an individual planet. However, exoplanets provide a statistical information over a wide range of mean densities which is not assessable for Solar System bodies. This statistical sample will significantly constrain future planet formation models and will lead to a better understanding of planet 
evolution. It is in this field where exoplanets probably will provide the largest impact.

Concerning water in a particular exoplanet, fortunately, water vapour can be measured directly in exoplanet atmospheres, as has been shown by recent detections of water in the atmospheres of close-in hot Jupiters using transmission spectroscopy, and in young gas giants at large orbital distances using high-contrast imaging. The detection of water in the atmosphere of terrestrial planets is however challenging and awaits future instrumentation.

Water is essential for life on Earth, and is often seen as necessary condition for the origin of life (Westall and Brack 2016; Cockell et al. 2016). Detecting water either indirectly (via mass and radius relationship) or directly (atmosphere spectroscopy) in exoplanets is therefore an important first step in the direction of finding life outside of the Solar System.

Water vapour in the atmosphere does not necessarily correlate with liquid water at the surface. Indeed, the past detections of water vapour in exoplanet atmospheres were only possible for hot Jupiters and one Neptune-like exoplanet, which would not be considered as good candidates for life (at least not Earth-like life). Even for smaller, terrestrial planets, water vapour does not need to refer to surface water, as can be seen in the case of Venus (e.g. Bullock and Grinspoon 2001).

On the other hand, liquid surface water also does not implicitly include habitability, as deep water layers (i.e. less-dense planets) may be uninhabitable (especially on massive planets, see Section 5.3). For the origin of life, the right amount of water matters.

In this paper we summarised the recent findings on the existence of water in exoplanets. The detection of low-density exoplanets gives an indirect detection of large amounts of water. Direct methods can be used to identify water in the atmosphere, and future missions will enable detection of water in the atmosphere of super-Earths (see Section 4), giving a clearer indication for the possible habitability of these planets. Ocean planets, hence planets without significant land masses, are shown to be less suitable for life due to negative $\mathrm{CO}_{2}$ feed-back (Kitzmann et al. 2015). Subsurface habitability for planets with large amounts of water is not necessarily ruled out, as even underneath deep ice layers life could flourish, as long as enough heat is produced in the rocky interior of the planet to melt the ice layer at the bottom. It will, however, be difficult to detect subsurface ocean life on exoplanets in the foreseeable future. Future missions therefore focus on the detection and characterisation of Earth-like planets which can be better compared to the evolution of life on our home planet.

Acknowledgements L.N. has been funded by the Interuniversity Attraction Poles Programme initiated by the Belgian Science Policy Office through the Planet Topers alliance. 


\section{References}

Y. Alibert, On the radius of habitable planets. $A \& A$ 561, 41 (2014)

G. Anglada-Escudé, B. Rojas-Ayala, A.P. Boss, A.J. Weinberger, J.P. Lloyd, Gj 1214 reviewed-trigonometric parallax, stellar parameters, new orbital solution, and bulk properties for the super-earth gj 1214b. A\&A 551, 48 (2013)

A. Baglin, M. Auvergne, L. Boisnard, T. Lam-Trong, P. Barge, C. Catala, M. Deleuil, E. Michel, W. Weiss, CoRoT: a high precision photometer for stellar ecolution and exoplanet finding, in 36th COSPAR Scientific assembly, vol. 36, 2006, p. 3749

T.S. Barman, Q.M. Konopacky, B. Macintosh, C. Marois, Simultaneous Detection of Water, Methane, and Carbon Monoxide in the Atmosphere of Exoplanet HR8799b. The Astrophys. J. 804, 61 (2015). doi:10.1088/0004-637X/804/1/61

N.M. Batalha, W.J. Borucki, S.T. Bryson, L.A. Buchhave, D.A. Caldwell, J. ChristensenDalsgaard, D. Ciardi, E.W. Dunham, F. Fressin, T.N. Gautier III, et al., Kepler's first rocky planet: Kepler-10bbased in part on observations obtained at the wm keck observatory, which is operated by the university of california and the california institute of technology. The Astrophysical Journal 729(1), 27 (2011)

J.L. Birkby, R.J. de Kok, M. Brogi, E.J.W. de Mooij, H. Schwarz, S. Albrecht, I.A.G. Snellen, Detection of water absorption in the day side atmosphere of HD $189733 \mathrm{~b}$ using ground-based high-resolution spectroscopy at $3.2 \mu \mathrm{m}$. Mon Not $R$ Astron Soc 436, 35-39 (2013). doi:10.1093/mnrasl/slt107

P.J. Boston, M.V. Ivanov, C.P. McKay, On the possibility of chemosynthetic ecosystems in subsurface habitats on mars. Icarus 95(2), 300-308 (1992)

C. Broeg, A. Fortier, D. Ehrenreich, Y. Alibert, W. Baumjohann, W. Benz, M. Deleuil, M. Gillon, A. Ivanov, R. Liseau, et al., CHEOPS: A transit photometry mission for ESA's small mission programme, in EPJ Web of Conferences, vol. 47, EDP Sciences, 2013, p. 3005. EDP Sciences

M. Brogi, R.J. de Kok, J.L. Birkby, H. Schwarz, I.A.G. Snellen, Carbon monoxide and water vapor in the atmosphere of the non-transiting exoplanet HD 179949 b. $A \& A \mathbf{5 6 5}, 124$ (2014). doi:10.1051/0004-6361/201423537

M.A. Bullock, D.H. Grinspoon, The recent evolution of climate on venus. Icarus 150, 19-37 (2001). doi:doi:10.1006/icar.2000.6570

J. Cabrera, H. Bruntt, M. Ollivier, R. Díaz, S. Csizmadia, S. Aigrain, R. Alonso, J.-M. Almenara, M. Auvergne, A. Baglin, et al., Transiting exoplanets from the corot space mission-xiii. corot-13b: a dense hot jupiter in transit around a star with solar metallicity and super-solar lithium content. $A \& A$ 522, $110(2010)$

A.C. Cameron, K. Horne, A. Penny, D. James, Probable detection of starlight reflected from the giant planet orbiting $\tau$ Boötis. Nature 402, 751-755 (1999). doi:10.1038/45451

D. Charbonneau, T.M. Brown, R.W. Noyes, R.L. Gilliland, Detection of an Extrasolar Planet Atmosphere. The Astrophys. J. 568, 377-384 (2002). doi:10.1086/338770

D. Charbonneau, L.E. Allen, S.T. Megeath, G. Torres, R. Alonso, T.M. Brown, R.L. Gilliland, D.W. Latham, G. Mandushev, F.T. O'Donovan, A. Sozzetti, Detection of Thermal Emission from an Extrasolar Planet. The Astrophys. J. 626, 523-529 (2005). doi:10.1086/429991

D. Charbonneau, H.A. Knutson, T. Barman, L.E. Allen, M. Mayor, S.T. Megeath, D. Queloz, S. Udry, The Broadband Infrared Emission Spectrum of the Exoplanet HD 189733b. The Astrophys. J. 686, 1341-1348 (2008). doi:10.1086/591635

D. Charbonneau, Z.K. Berta, J. Irwin, C.J. Burke, P. Nutzman1, et al., A super-Earth transiting a nearby low-mass star. Nature 462, 891-894 (2009)

C. Cockell, T. Bush, C. Bryce, S. Direito, M. Fox-Powell, J. Harrison, H. Lammer, H. Landenmark, J. Martin-Torres, N. Nicholson, et al., Habitability: A review. Astrobiology 16(1), 89-117 (2016)

T. Currie, P. Hinz, Y. Itoh, M. Fukagawa, V. Bailey, T.J. Rodigas, D. Fabrycky, R. MurrayClay, Direct imaging of the brown dwarf/planet-mass companions to HR 8799 and GJ 758 from 1 to 5 microns: Constraints on atmospheric properties, in In the Spirit of Lyot 2010, 2010

C. Darwin, On the origin of species. John Murray, London, 490 (1859) 
M. Deleuil, A. Bonomo, S. Ferraz-Mello, A. Erikson, F. Bouchy, M. Havel, S. Aigrain, J.-M. Almenara, R. Alonso, M. Auvergne, et al., Transiting exoplanets from the corot space mission-xx. corot-20b: A very high density, high eccentricity transiting giant planet. Astronomy \& Astrophysics 538, 145 (2012)

D. Deming, S. Seager, L.J. Richardson, J. Harrington, Infrared radiation from an extrasolar planet. Nature 434, 740-743 (2005). doi:10.1038/nature03507

D. Deming, A. Wilkins, P. McCullough, A. Burrows, J.J. Fortney, E. Agol, I. Dobbs-Dixon, N. Madhusudhan, N. Crouzet, J.-M. Desert, R.L. Gilliland, K. Haynes, H.A. Knutson, M. Line, Z. Magic, A.M. Mandell, S. Ranjan, D. Charbonneau, M. Clampin, S. Seager, A.P. Showman, Infrared Transmission Spectroscopy of the Exoplanets HD 209458b and XO-1b Using the Wide Field Camera-3 on the Hubble Space Telescope. The Astrophys. J. 774, 95 (2013). doi:10.1088/0004-637X/774/2/95

C. Dorn, A. Khan, K. Heng, J.A.D. Connolly, Y. Alibert, W. Benz, P. Tackley, Can we constrain the interior structure of rocky exoplanets from mass and radius measurements? A\&A 577(183), 1-18 (2015)

C.D. Dressing, D. Charbonneau, X. Dumusque, et al., The Mass of Kepler-93b and The Composition of Terrestrial Planets. The Astrophys. J. 800(2), 135 (2015)

A.M. Dziewonski, D.L. Anderson, Preliminary reference Earth model. Phys Earth Planet In 25(4), 297-356 (1981)

B.J. Foley, The role of plate tectonic-climate coupling and exposed land area in the development of habitable climates on rocky planets. The Astrophysical Journal 812(1), 36 (2015)

J. Fraine, D. Deming, B. Benneke, H. Knutson, A. Jordán, N. Espinoza, N. Madhusudhan, A. Wilkins, K. Todorov, Water vapour absorption in the clear atmosphere of a Neptunesized exoplanet. Nature 513, 526-529 (2014). doi:10.1038/nature13785

Y. Fujii, H. Kawahara, Y. Suto, A. Taruya, S. Fukuda, T. Nakajima, E.L. Turner, Colors of a second earth: Estimating the fractional areas of ocean, land, and vegetation of earth-like exoplanets. The Astrophys. J. 715(2), 866 (2010)

T. Gold, The deep, hot biosphere. PNAS 89(13), 6045-6049 (1992)

O. Grasset, J. Schneider, C. Sotin, A study of the accuracy of massradius relationships for silicate-rich and ice-rich planets up to 100 earth masses. The Astrophys. J. 693, 722-733 (2009)

C.J. Grillmair, A. Burrows, D. Charbonneau, L. Armus, J. Stauffer, V. Meadows, J. van Cleve, K. von Braun, D. Levine, Strong water absorption in the dayside emission spectrum of the planet HD189733b. Nature 456, 767-769 (2008). doi:10.1038/nature07574

P.M. Grindrod, A.D. Fortes, F. Nimmo, D.L. Feltham, J.P. Brodholt, L. Vocadlo, The longterm stability of a possible aqueous ammonium sulfate ocean inside Titan. Icarus 197, 137-151 (2008)

I. Halevy, J.W. Head III, Episodic warming of early mars by punctuated volcanism. Nature Geoscience 7(12), 865-868 (2014)

M.H. Hart, Habitable Zones about Main Sequence Stars. Icarus 37, 351-357 (1979). doi:10.1016/0019-1035(79)90141-6

A.P. Hatzes, H. Rauer, A definition for giant planets based on the mass-density relationship. The Astrophys. J. Letters $\mathbf{8 1 0}(2), 25$ (2015)

J. Helbert, E. Hauber, D. Reiss, Water on the Terrestrial Planets, in Treatise on Geophysics (Second Edition), Volume 10: Physics of Terrestrial Planets and Moons (Springer, Dordrecht, 2015), pp. 367-409. doi:10.1016/B978-0-444-53802-4.00174-3

C.E. Hinchliff, et al., Synthesis of phylogeny and taxonomy into a comprehensive tree of life. PNAS 112(41), 12764-12769 (2015). doi:10.1073/pnas.1423041112

D. Höning, H. Hansen-Goos, A. Airo, T. Spohn, Biotic vs. abiotic earth: A model for mantle hydration and continental coverage. Planet Space Sci 98, 5-13 (2014)

S.B. Howell, C. Sobeck, M. Haas, M. Still, T. Barclay, F. Mullally, J. Troeltzsch, S. Aigrain, S.T. Bryson, D. Caldwell, et al., The k2 mission: Characterization and early results. Publications of the Astronomical Society of the Pacific 126(938), 398 (2014)

L. Iess, R.A. Jacobson, M. Ducci, D.J. Stevenson, J.I. Lunine, J.W. Armstrong, S.W. Asmar, P. Racioppa, N.J. Rappaport, P. Tortora, The Tides of Titan. Science 337(6093), 457459 (2012). doi:10.1126/science.1219631

J.M. Jenkins, J.D. Twicken, N.M. Batalha, D.A. Caldwell, W.D. Cochran, M. Endl, D.W. 
Latham, G.A. Esquerdo, S. Seader, A. Bieryla, et al., Discovery and validation of kepler452b: A $1.6 \mathrm{r}$ ? super earth exoplanet in the habitable zone of a g2 star. The Astronomical Journal 150(2), $56(2015)$

J.F. Kasting, D.P. Whitmire, R.T. Reynolds, Habitable Zones around Main Sequence Stars. Icarus 101, 108-128 (1993). doi:10.1006/icar.1993.1010

K. Khurana, M. Kivelson, D. Stevenson, G. Schubert, C. Russell, R. Walker, C. Polanskey, Induced magnetic fields as evidence for subsurface oceans in Europa and Callisto. Nature 395(6704), 777-780 (1998)

D. Kitzmann, Y. Alibert, M. Godolt, J.L. Grenfell, K. Heng, A. Patzer, H. Rauer, B. Stracke, P. von Paris, The unstable $\mathrm{CO}_{2}$ feedback cycle on ocean planets. Mon Not $R$ Astron Soc 452(4), 3752-3758 (2015)

M. Kivelson, K. Khurana, M. Volwerk, The permanent and inductive magnetic moments of Ganymede. Icarus 157(2), 507-522 (2002)

H.A. Knutson, D. Charbonneau, L.E. Allen, J.J. Fortney, E. Agol, N.B. Cowan, A.P. Showman, C.S. Cooper, S.T. Megeath, A map of the day-night contrast of the extrasolar planet HD 189733b. Nature 447, 183-186 (2007). doi:10.1038/nature05782

H.A. Knutson, D. Dragomir, L. Kreidberg, E.M.-R. Kempton, P.R. McCullough, J.J. Fortney, J.L. Bean, M. Gillon, D. Homeier, A.W. Howard, Hubble Space Telescope Near-IR Transmission Spectroscopy of the Super-Earth HD 97658b. The Astrophys. J. 794, 155 (2014). doi:10.1088/0004-637X/794/2/155

D.G. Koch, W.J. Borucki, G. Basri, N.M. Batalha, T.M. Brown, D. Caldwell, J. ChristensenDalsgaard, W.D. Cochran, E. DeVore, E.W. Dunham, et al., Kepler mission design, realized photometric performance, and early science. The Astrophysical Journal Letters $\mathbf{7 1 3}(2), 79(2010)$

Q.M. Konopacky, T.S. Barman, B.A. Macintosh, C. Marois, Detection of Carbon Monoxide and Water Absorption Lines in an Exoplanet Atmosphere. Science 339, 1398-1401 (2013). doi:10.1126/science.1232003

R.K. Kopparapu, R. Ramirez, J.F. Kasting, V. Eymet, T.D. Robinson, S. Mahadevan, R.C. Terrien, S. Domagal-Goldman, V. Meadows, R. Deshpande, Habitable Zones around Main-sequence Stars: New Estimates. The Astrophys. J. 765, 131 (2013). doi:10.1088/0004-637X/765/2/131

L. Kreidberg, J.L. Bean, J.-M. Désert, M.R. Line, J.J. Fortney, N. Madhusudhan, K.B. Stevenson, A.P. Showman, D. Charbonneau, P.R. McCullough, S. Seager, A. Burrows, G.W. Henry, M. Williamson, T. Kataria, D. Homeier, A Precise Water Abundance Measurement for the Hot Jupiter WASP-43b. The Astrophys. J. Letters 793, 27 (2014a). doi:10.1088/2041-8205/793/2/L27

L. Kreidberg, J.L. Bean, J.-M. Désert, B. Benneke, D. Deming, K.B. Stevenson, S. Seager, Z. Berta-Thompson, A. Seifahrt, D. Homeier, Clouds in the atmosphere of the super-Earth exoplanet GJ1214b. Nature 505, 69-72 (2014b). doi:10.1038/nature12888

M.J. Kuchner, Volatile-rich Earth-mass planets in the habitable zone. The Astrophys. J. 596, 105-108 (2003)

A.-M. Lagrange, M. Bonnefoy, G. Chauvin, D. Apai, D. Ehrenreich, A. Boccaletti, D. Gratadour, D. Rouan, D. Mouillet, S. Lacour, M. Kasper, A Giant Planet Imaged in the Disk of the Young Star $\beta$ Pictoris. Science 329, 57 (2010). doi:10.1126/science.1187187

H. Lammer, J.H. Bredehöft, A. Coustenis, et al., What makes a planet habitable? A\&A 17, 181-249 (2009)

H. Lammer, A. Stökl, N.V. Erkaev, E.A. Dorfi, P. Odert, et al., Origin and loss of nebulacaptured hydrogen envelopes from sub- to super-Earths in the habitable zone of Sun-like stars. Mon Not R Astron Soc 439(4), 3225-3238 (2014)

H. Lammer, N. Erkaev, L. Fossati, I. Juvan, P. Odert, P. Cubillos, E. Guenther, K. Kislyakova, C. Johnstone, T. Lüftinger, et al., Identifying the "true" radius of the hot sub-neptune corot-24b by mass loss modelling. Mon Not R Astron Soc 461(1, slw095), 62-66 (2016)

G. Laughlin, J.J. Lissauer, Exoplanetary geophysics-an emerging discipline. arXiv preprint arXiv:1501.05685 (2015)

A. Léger, F. Selsis, C. Sotin, et al., A new family of planets? 'Ocean-Planets'. Icarus 169(2), 499-504 (2004)

A. Léger, D. Rouan, J. Schneider, P. Barge, M. Fridlund, et al., Transiting exoplanets from 
the CoRoT space mission. VIII. CoRoT-7b: the first super-Earth with measured radius. A\&A 506, 287-302 (2009). doi:10.1051/0004-6361/200911933

J.J. Lissauer, D.C. Fabrycky, E.B. Ford, et al., A closely packed system of low-mass, low-density planets transiting Kepler-11. Nature 470, 53-58 (2011). doi:10.1038/nature09760

A.C. Lockwood, J.A. Johnson, C.F. Bender, J.S. Carr, T. Barman, A.J.W. Richert, G.A. Blake, Near-IR Direct Detection of Water Vapor in Tau Boötis b. The Astrophys. J. Letters 783, 29 (2014). doi:10.1088/2041-8205/783/2/L29

R. Luger, R. Barnes, E. Lopez, J. Fortney, B. Jackson, V. Meadows, Habitable Evaporated Cores: Transforming Mini-Neptunes into Super-Earths in the Habitable Zones of M Dwarfs. Astrobiology 15, 57-88 (2015). doi:10.1089/ast.2014.1215

A.M. Mandell, K. Haynes, E. Sinukoff, N. Madhusudhan, A. Burrows, D. Deming, Exoplanet Transit Spectroscopy Using WFC3: WASP-12 b, WASP-17 b, and WASP-19 b. The Astrophys. J. 779, 128 (2013). doi:10.1088/0004-637X/779/2/128

G.W. Marcy, R.P. Butler, A Planetary Companion to 70 Virginis. The Astrophys. J. Letters 464, 147 (1996). doi:10.1086/310096

G.W. Marcy, H. Isaacson, A.W. Howard, J.F. Rowe, J.M. Jenkins, et al., Masses, radii, and orbits of small Kepler planets: The transition from gaseous to rocky planets. The Astrophysical Journal Supplement Series 210, 20-70 (2014)

C. Marois, B. Macintosh, T. Barman, B. Zuckerman, I. Song, J. Patience, D. Lafrenière, R. Doyon, Direct Imaging of Multiple Planets Orbiting the Star HR 8799. Science 322, 1348 (2008). doi:10.1126/science.1166585

S. Maruyama, M. Ikoma, H. Genda, et al., The naked planet Earth: Most essential prerequisite for the origin and evolution of life. Geoscience Frontiers 4, 141-165 (2013)

M. Mayor, D. Queloz, A Jupiter-Mass Companion to a Solar-Type Star. Nature 378, 355 (1995). doi:10.1038/378355a0

P.R. McCullough, N. Crouzet, D. Deming, N. Madhusudhan, Water Vapor in the Spectrum of the Extrasolar Planet HD 189733b. I. The Transit. The Astrophys. J. 791, 55 (2014). doi:10.1088/0004-637X/791/1/55

C.P. McKay, W.L. Davis, Duration of liquid water habitats on early Mars. Icarus 90(2), 214-221 (1991)

J. Milli, D. Mouillet, D. Mawet, H.M. Schmid, A. Bazzon, J.H. Girard, K. Dohlen, R. Roelfsema, Prospects of detecting the polarimetric signature of the Earth-mass planet $\alpha$ Centauri B b with SPHERE/ZIMPOL. A\&A 556, 64 (2013). doi:10.1051/0004$6361 / 201321881$

P. Montanes-Rodriguez, E. Palle, P.R. Goode, F.J. Martin-Torres, Vegetation signature in the observed globally integrated spectrum of earth considering simultaneous cloud data: Applications for extrasolar planets. The Astrophys. J. 651(1), 544 (2006)

C. Mordasini, Y. Alibert, H. Klahr, T. Henning, Characterization of exoplanets from their formation-i. models of combined planet formation and evolution. Astronomy \& Astrophysics 547, $111(2012 \mathrm{a})$

C. Mordasini, Y. Alibert, W. Benz, H. Klahr, T. Henning, Extrasolar planet population synthesis-iv. correlations with disk metallicity, mass, and lifetime. Astronomy \& Astrophysics 541, 97 (2012b)

N. Nettelmann, J. Fortney, U. Kramm, R. Redmer, Thermal evolution and structure models of the transiting super-earth gj 1214b. The Astrophysical Journal 733(1), 2 (2011)

L. Noack, D. Breuer, Interior and surface dynamics of terrestrial bodies and their implications for the habitability, in Habitability on other planets and satellites: The quest for extraterrestrial life, ed. by J.-P. de Vera, F. Seckbach (Springer, Dordrecht, 2013), pp. 203-233

L. Noack, D. Breuer, Plate tectonics on rocky exoplanets: Influence of initial conditions and rheology. Planet Space Sci 98, 41-49 (2014). doi:10.1016/j.pss.2013.06.020

L. Noack, M. Godolt, P. von Paris, A. Plesa, B. Stracke, D. Breuer, H. Rauer, Constraints for planetary habitability from interior modeling. Planet Space Sci 98, 14-29 (2014)

L. Noack, D. Höning, A. Rivoldini, C. Heistracher, N. Zimov, B. Journaux, H. Lammer, T.V. Hoolst, J.H. Bredehöft, Water-rich planets: how habitable is a water layer deeper than on Earth? Icarus 277, 215-236 (2016). doi:10.1016/j.icarus.2016.05.009

L. Noack, D. Höning, A. Rivoldini, C. Heistracher, N. Zimov, B. Journaux, H. Lammer, T. 
Van Hoolst, J.H. Bredehöft, Water-rich planets: How habitable is a water layer deeper than on earth? Icarus 277, 215-236 (2017)

R. Parai, S. Mukhopadhyay, How large is the subducted water flux? new constraints on mantle regassing rates. EPSL 317, 396-406 (2012)

S. Ramsay, M. Casali, J. González, N. Hubin, The E-ELT instrument roadmap: a status report, in SPIE Astronomical Telescopes + Instrumentation, International Society for Optics and Photonics, 2014, pp. 91471-10. International Society for Optics and Photonics

H. Rauer, C. Catala, C. Aerts, et al., The PLATO 2.0 mission. Experimental Astronomy 38(1-2), 249-330 (2014). doi:10.1007/s10686-014-9383-4

P. Riaud, J. Schneider, Improving Earth-like planets' detection with an ELT: the differential radial velocity experiment. A\&SA 469, 355-361 (2007). doi:10.1051/0004-6361:20077085

G.R. Ricker, J.N. Winn, R. Vanderspek, D.W. Latham, G.Á. Bakos, et al., Transiting exoplanet survey satellite (tess). Proceedings of the SPIE 9143(914320), 15 (2014)

G.R. Ricker, J.N. Winn, R. Vanderspek, D.W. Latham, G.Á. Bakos, J.L. Bean, Z.K. BertaThompson, T.M. Brown, L. Buchhave, N.R. Butler, et al., Transiting exoplanet survey satellite. Journal of Astronomical Telescopes, Instruments, and Systems 1(1), 014003014003 (2015)

L. Rogers, S. Seager, Three possible origins for the gas layer on gj 1214b. The Astrophysical Journal 716(2), 1208 (2010)

L.A. Rogers, Most 1.6 Earth-radius Planets are Not Rocky. The Astrophys. J. 801(1), 41 (2015)

L. Roth, J. Saur, K.D. Retherford, D.F. Strobel, P.D. Feldman, M.A. McGrath, F. Nimmo, Transient water vapor at europas south pole. Science 343(6167), 171-174 (2014)

P.A. Sabelhaus, J.E. Decker, An overview of the James Webb Space Telescope (JWST) project, in SPIE Astronomical Telescopes+ Instrumentation, International Society for Optics and Photonics, 2004, pp. 550-563. International Society for Optics and Photonics

N.C. Santos, V. Adibekyan, C. Mordasini, W. Benz, E. Delgado-Mena, C. Dorn, L. Buchhave, P. Figueira, A. Mortier, F. Pepe, A. Santerne, S.G. Sousa, S. Udry, Constraining planet structure from stellar chemistry: the cases of CoRoT-7, Kepler-10, and Kepler-93. $A \& A$ 580(L13), 1-5 (2015)

J. Saur, S. Duling, L. Roth, X. Jia, D.F. Strobel, P.D. Feldman, U.R. Christensen, K.D. Retherford, M.A. McGrath, F. Musacchio, et al., The search for a subsurface ocean in Ganymede with Hubble Space Telescope observations of its auroral ovals. Journal of Geophysical Research: Space Physics 120(3), 1715-1737 (2015)

L. Schaefer, D. Sasselov, The persistence of oceans on earth-like planets: insights from the deep-water cycle. The Astrophys. J. 801(1), 40 (2015)

G. Schubert, D.L. Turcotte, P. Olson, Mantle convection in the Earth and planets (Cambridge University Press, New York, 2001)

H. Schwarz, M. Brogi, R. de Kok, J. Birkby, I. Snellen, Measuring the spin of the directly imaged sub-stellar companion GQ Lupi b, in AAS/Division for Extreme Solar Systems Abstracts. AAS/Division for Extreme Solar Systems Abstracts, vol. 3, 2015, pp. 104-14

S. Seager, Exoplanet Habitability. Science 340, 577-581 (2013)

D.K. Sing, J.J. Fortney, N. Nikolov, H.R. Wakeford, T. Kataria, T.M. Evans, S. Aigrain, G.E. Ballester, A.S. Burrows, D. Deming, J.-M. Désert, N.P. Gibson, G.W. Henry, C.M. Huitson, H.A. Knutson, A.L.D. Etangs, F. Pont, A.P. Showman, A. Vidal-Madjar, M.H. Williamson, P.A. Wilson, A continuum from clear to cloudy hot-Jupiter exoplanets without primordial water depletion. Nature 529, 59-62 (2016). doi:10.1038/nature16068

W. Skidmore, TMT International Science Development Teams, TMT Science Advisory Committee, Thirty meter telescope detailed science case: 2015. Res Astron Astrophys 15(12), 1945 (2015). http://stacks.iop.org/1674-4527/15/i=12/a=1945

I.A.G. Snellen, R.J. de Kok, E.J.W. de Mooij, S. Albrecht, The orbital motion, absolute mass and high-altitude winds of exoplanet HD209458b. Nature 465, 1049-1051 (2010). doi:10.1038/nature09111

I.A.G. Snellen, B.R. Brandl, R.J. de Kok, M. Brogi, J. Birkby, H. Schwarz, Fast spin of the young extrasolar planet $\beta$ Pictoris b. Nature 509, 63-65 (2014). doi:10.1038/nature13253 
I. Snellen, R. de Kok, J.L. Birkby, B. Brandl, M. Brogi, C. Keller, M. Kenworthy, H. Schwarz, R. Stuik, Combining high-dispersion spectroscopy with high contrast imaging: Probing rocky planets around our nearest neighbors. A\&A 576, 59 (2015). doi:10.1051/0004$6361 / 201425018$

C. Sotin, O. Grasset, A. Mocquet, Massradius curve for extrasolar Earth-like planets and ocean planets. Icarus 191(1), 337-351 (2007)

W.B. Sparks, H.C. Ford, Imaging Spectroscopy for Extrasolar Planet Detection. The Astrophys. J. 578, 543-564 (2002). doi:10.1086/342401

V. Stamenković, D. Breuer, The tectonic mode of rocky planets: Part 1-Driving factors, models \& parameters. Icarus 234, 174-193 (2014)

C. Stein, J.P. Lowman, U. Hansen, The influence of mantle internal heating on lithospheric mobility: implications for super-Earths. EPSL 361, 448-459 (2013)

D.J. Stevenson, Life-sustaining planets in interstellar space? Nature 400(6739), 32-32 (1999)

M.R. Swain, G. Vasisht, G. Tinetti, The presence of methane in the atmosphere of an extrasolar planet. Nature 452, 329-331 (2008). doi:10.1038/nature06823

P. Thomas, R. Tajeddine, M. Tiscareno, J. Burns, J. Joseph, T. Loredo, P. Helfenstein, C. Porco, Enceladuss measured physical libration requires a global subsurface ocean. Icarus 264, 37-47 (2016)

G. Tinetti, A. Vidal-Madjar, M.-C. Liang, J.-P. Beaulieu, Y. Yung, S. Carey, R.J. Barber, J. Tennyson, I. Ribas, N. Allard, G.E. Ballester, D.K. Sing, F. Selsis, Water vapour in the atmosphere of a transiting extrasolar planet. Nature 448, 169-171 (2007). doi: $10.1038 /$ nature06002

C.T. Unterborn, E.E. Dismukes, W.R. Panero, Scaling the Earth: A Sensitivity Analysis of Terrestrial Exoplanetary Interior Models. The Astrophys. J. 819(1), 32 (2016)

D. Valencia, R.J. O'Connell, D.D. Sasselov, Inevitability of Plate Tectonics on Super-Earths. The Astrophys. J. 670, 45-48 (2007)

D. Valencia, R.J. O'Connell, D.D. Sasselov, Internal structure of massive terrestrial planets. Icarus 181, 545-554 (2006)

D. Valencia, T. Guillot, V. Parmentier, R.S. Freedman, Bulk composition of gj 1214b and other sub-neptune exoplanets. The Astrophysical Journal 775(1), 10 (2013)

H.J. van Heck, P.J. Tackley, Plate tectonics on super-Earths: Equally or more likely than on Earth. EPSL 310, 252-261 (2011)

T. Van Hoolst, R.-M. Baland, A. Trinh, The diurnal libration and interior structure of Enceladus. Icarus 277, 311-318 (2016)

S. Vance, M. Bouffard, M. Choukroun, C. Sotin, Ganymede's internal structure including thermodynamics of magnesium sulfate oceans in contact with ice. Planet Space Sci 96, 62-70 (2014)

S. Vance, K. Hand, R. Pappalardo, Geophysical controls of chemical disequilibria in Europa. GRL (2016)

F.W. Wagner, N. Tosi, F. Sohl, H. Rauer, T. Spohn, Rocky super-Earth interiors structure and internal dynamics of CoRoT-7b and Kepler-10b. A\&A 541, 103 (2012)

H.R. Wakeford, D.K. Sing, D. Deming, N.P. Gibson, J.J. Fortney, A.S. Burrows, G. Ballester, N. Nikolov, S. Aigrain, G. Henry, H. Knutson, A. Lecavelier des Etangs, F. Pont, A.P. Showman, A. Vidal-Madjar, K. Zahnle, HST hot Jupiter transmission spectral survey: detection of water in HAT-P-1b from WFC3 near-IR spatial scan observations. Mon Not R Astron Soc 435, 3481-3493 (2013). doi:10.1093/mnras/stt1536

J.C.G. Walker, P.B. Hays, J.F. Kasting, A negative feedback mechanism for the long-term stabilization of the earth's surface temperature. J. Geophys. Res. 86, 9776-9782 (1981)

C.R. Webster, P.R. Mahaffy, S.K. Atreya, G.J. Flesch, M.A. Mischna, P.-Y. Meslin, K.A. Farley, P.G. Conrad, L.E. Christensen, A.A. Pavlov, et al., Mars methane detection and variability at Gale crater. Science 347, 415-417 (2015). doi:10.1126/science.1261713

F. Westall, A. Brack, The importance of water for life. Space Sci. Rev. submitted (this issue) (2016)

A. Wolszczan, D.A. Frail, A planetary system around the millisecond pulsar psr1257 +12 . Nature 355, 145-147 (1992)

C. Zimmer, K.K. Khurana, M.G. Kivelson, Subsurface oceans on Europa and Callisto: Constraints from Galileo magnetometer observations. Icarus 147(2), 329-347 (2000) 